\title{
Disorderly Molecules and Refrigerating Engineering*
}

$\mathrm{T}$ HE many facets of the jewel of truth are only too often exposed in a sequence that is bewildering but, even so, it is not often that succeeding facets reflect such dissimilar beams as at first sight to seem to demand the ascription of conflicting virtues to the body of the jewel behind. Sir William Bragg, giving the James Forrest Lecture last year, dealt with the orderly arrangement of the atoms within crystals: Sir Frank Smith, the lecturer for this year, was concerned to demonstrate how the disorderly or random nature of the molecular motions within a substance make it possible for chemical actions on one hand, and the refrigeration industry on the other hand, to exist.

Accepting the identity of the motion of molecules with heat and the corollary that the temperature of a body is a measure of the intensity of their translatory movement, Sir Frank pointed out, in illustration, that if at a given moment the molecules in the air in the lecture theatre were all to be in simultaneous collision with each other, the temperature of the air would drop to absolute zero. Incidentally, under the influence of the gravitational pull, they would fall to the floor. With his assertion that the attainment of very low temperatures has been one of the great scientific advances in recent years, few-and certainly no engineer-would disagree: the knowledge gained of the motion of liquid molecules and of the evaporation of liquids has found widespread application at more elevated temperatures in many fields, of which refrigerating engineering in particular is the obvious example.

Sir Frank Smith purposely refrained from embarking on a historical résumé of low-temperature work, and from employing a mathematical treatment or the empiricisms of Boyle's Law or its various refinements to explain the phenomena he was considering. Instead, he tried to make his approach solely from considerations of the general nature of atoms and molecules, their movements and their interactions. Assuming only that atoms consist of charged nuclei surrounded by electron shells, he proceeded to consider in general terms what might be expected to happen in a collision between two similar atoms. The collision would inevitably set up some, probably electromagnetic, stress and if the collision were such that, for a moment of time, both were brought to a standstill, then the kinetic energies of each of the atoms would

* Account of the James Forrest Lecture delivered by Sir Frank Smith, K.C.B.,F.R.S., before the Institution of Civil Engineers on May 3. disappear and the electromagnetic stresses would simultaneously reach their maximum values. Afterwards, the stressed systems would cause repulsion to take place and the atoms would then move away from one another. However, in addition to this force of repulsion effective at distances comparable with molecular diameters, there is a general force of attraction between atoms and molecules which he suggested might be of the nature of electromagnetic induction. These forces and their resultant were shown graphically, plotting nuclear distances against the force exerted, and it was shown that the curve embraces the solid, liquid and gaseous states and that it indicates that increase in the temperature of a substance will cause expansion. The slope of the curve at zero force is a measure of the elasticity of the material, and the maximum value of the 'resultant' force indicates the maximum force which can be applied without rupture to the substance in the solid form. The value of the nuclear distance at this point represents the ideal elastic limit of the substance; but, in practice, factors introduced by the presence of impurities or other causes modify the attainable elastic limit and rupturing force.

Attention was then directed to a consideration of the Joule-Thomson effect. In every gas there are two effects on expansion, one tending to lower and the other to raise the temperature. The cooling effect depends on the degree of separation of the molecules, and the heating effect depends on molecular collisions, increasing with increase of temperature. At sufficiently high temperatures the net effect is to produce a rise in temperature, at the inversion point there is no change and below it there is a fall in temperature on expansion. Liquefaction of air by compression alone is im. possible at ordinary temperatures, and preliminary cooling to below the critical temperature is necessary, although by the use of a cold regenerator or heat-exchanger the need for very high pressures and very low initial temperatures is made unnecessary.

After a brief explanation of the Claude expansion method of refrigeration, Sir Frank Smith passed to the production of relatively pure atmospheric gases by rectification (or the separation of the various constituents of liquid air by making use of their different boiling points) and indicated various commercial uses of the gases so obtained. The same method is applied to water gas and coke- 
oven gas and can be, and sometimes is, used commercially to produce relatively pure hydrogen.

These methods have been used under suitable conditions to attain temperatures less than $1^{\circ} \mathrm{K}$., but, while it is admitted that it is most improbable that the absolute zero of temperature will ever be reached, advantage has been taken of the magnetic properties of matter to approach it very closely. The phenomena we know as magnetism are due to the existence of atomic magnetic dipoles which, in a magnetic field, are pulled in the direction of that field. The motion of the atoms which is associated with heat resists this pull, and so the resultant magnetic field depends on the temperature as well as on the intensity of the applied magnetic field: the higher the temperature, the less the magnetization. In general, the magnetic dipoles become arranged in orderly fashion at comparatively high temperatures, but there are a few substances, such as iron ammonium alum, in which random orientation still exists at very low temperatures. When such substances are at the lowest temperature attainable by the methods already described, application of a magnetic field modifies the dipole directions and heat is developed. This heat is slowly absorbed by the cold surrounding surfaces, and when the original low temperature has been restored the magnetic field is removed. The dipoles then tend to revert to their previous random orientation and, in so doing, absorb heat, so that the temperature drops. By making use of this effect, a temperature as low as $0.003^{\circ} \mathrm{K}$. has been reached.

Turning back from very low temperatures, a pause was made to consider in some detail the mechanism of the evaporation of liquids. Maxwell's law of distribution shows that at any time some molecules are moving with a velocity ten or twenty times greater than that of others. Such fastmoving molecules are the ones which escape from a liquid, and in so doing they tend to lower the average velocity, and hence the temperature, of the remainder. Equally, it is the fast-moving molecules of vapour which tend to re-enter the liquid and increase the average velocity of the molecules there. This counter-effect produces a heating of the liquid. The actual temperature of the liquid will thus be determined by an algebraic summation of these two effects and in order, on balance, to produce cooling it is necessary to eliminate the 'counter-effect' by removing the molecules of vapour as soon as they leave the liquid. By this device, quite low temperatures may be attained.

This effect is the basis of most modern commercial refrigeration machines. Even a century ago, Faraday reached a temperature of $160^{\circ} \mathrm{K}$. by evaporating a liquid under an air-pump and to-day, by using helium, a temperature of $1^{\circ} \mathrm{K}$. may be attained. Present refrigerating practice utilizes substances (principally ammonia and carbon dioxide) which are vapours at ordinary temperatures and pressures and can be liquefied by subjection to increase of pressure but without preliminary cooling: atmospheric temperature is well below their critical temperatures. It is necessary, therefore, that any pumping mechanism should not only exhaust the vapours from the upper surface of the liquid but should also be able to compress them. When compressed, the gas molecules are brought closer together and the heat produced is extracted by cold water surrounding the compression chamber. The attractive force between the gas molecules becomes greater and greater as the pressure is increased and eventually the substance liquefies. This cold liquid is passed through an expansion- or throttling-valve, and at the same time the pressure to which the liquid is subjected on emergence from the valve is so reduced by suction that the liquid boils. Brine which is circulating around this chamber is therefore cooled and then passes through pipes and tanks as may be desired. It is part of the process to collect the vapours removed from the expansion chamber and to recompress them so that they undergo a continuous process of compression and expansion, liquefaction and evaporization.

Refrigeration engineering now finds application not only to the production of ice and, extensively, to the transport and storage of food of many kinds, but also to the cold storage of bulbs, the conditioning of air for public rooms, cold depositories for furs, carpets and many textile goods, the production of ice in skating-rinks and the freezing of ground for shaft-sinking and tunnelling in water-logged strata.

Whereas, sixty years ago, there was no refrigerating machine and certainly no cold storage provided in ships, to-day the refrigerated space in ships bringing food to Great Britain alone amounts to not less than 100 million cubic feet, equivalent-as it was rather strikingly put-to a single cold store as wide as Victoria Street, London, 20 feet high and 13 miles long. No fewer than thirty countries contribute to our food supply by the help of refrigeration, and it may truly be said that our food can now be practically independent of the seasons.

In very low temperature work, however, progress has not been so rapid, and Sir Frank Smith confessed to a hope that engineers might be persuaded to take an active interest in the problems involved, and commended to his audience the work in progress in the laboratories at both Oxford and Cambridge.

H. W. 